\title{
A LARGE-DEFLECTION ELECTROSTATIC ACTUATOR FOR OPTICAL SWITCHING APPLICATIONS
}

\author{
John D. Grade ${ }^{*}$ and Hal Jerman \\ Seagate Research \\ San Jose, CA 95131 \\ Thomas W. Kenny \\ Department of Mechanical Engineering, Stanford University \\ Stanford, CA 94305
}

\begin{abstract}
Fundamental improvements in the design of comb-drive actuators have allowed their use in a $1 \mathrm{xN}$ optical switch requiring 200 um static deflection and a switching speed of less than $1 \mathrm{~ms}$. The main obstacle to the development of large-displacement combdrive actuators has been electromechanical side instability, which increases with forward deflection and may cause catastrophic failure. In this work, the stable deflection range is increased by employing prebent suspensions and linearly engaging comb teeth. Nonlinear analytical models, as well as Mechanica ${ }^{\mathrm{TM}}$ finite element models and Simulink ${ }^{\mathrm{TM}}$ numerical models have been developed to fully characterize the performance of the actuators and facilitate their design. A simple, high-yield, deep-reactive-ionetching fabrication process has produced consistent performance that closely matches FEA and numerical models of the electrostatic actuators. Using separately-fabricated, highly-reflective mirrors, the actuators have becn utilized in a compact optical design that features high-speed, low optical-loss switching.
\end{abstract}

\section{INTRODUCTION}

There has been considerable interest in devices for switching light from one optical fiber to another or from a free-space optical beam to one or more optical fibers, particularly for telecommunications. Several switch configurations are of interest, including $1 \times 2,1 \times N$, and $N x N$, where $N$ is a number from 2 to above 64 . The dominant optical switch designs have utilized optical waveguides, electro-optical effects, or electromechanical actuators, and working switches are commercially available using these techniques.

Of the $1 \times \mathrm{N}$ electromechanical optical switches, the most common design uses a single rotary actuator to "steer" the optical beam to a particular output location. An alternate switch architecture uses multiple linear actuators to intercept and redirect the optical beam into a selected output fiber [1]. Figure 1 shows an example of such a switch with four 45 degree mirrors attached to independent linear actuators, each capable of diverting the collimated beam to a particular output. While this switch architecture does not require the precision servo-control of the beam-steering designs, it does require each linear actuator to travel at least the diameter of the collimated beam, which is greater than $100 \mathrm{um}$ for practical optical layouts.

Traditional designs for linear comb drive actuators show limited forward displacement due to electromechanical side instability. Side instability occurs when the parallel-plate electrostatic forces between the sides of the combs overwhelm the suspension stiffness orthogonal to the desired motion [2-5]. This problem is exacerbated during large displacements, because with increasing forward displacement, a traditional folded-beam suspension's side stiffness decreases while the electrostatic side forces increase.

*e-mail: John_D_Grade@notes.seagate.com Phone: (408)952-5036

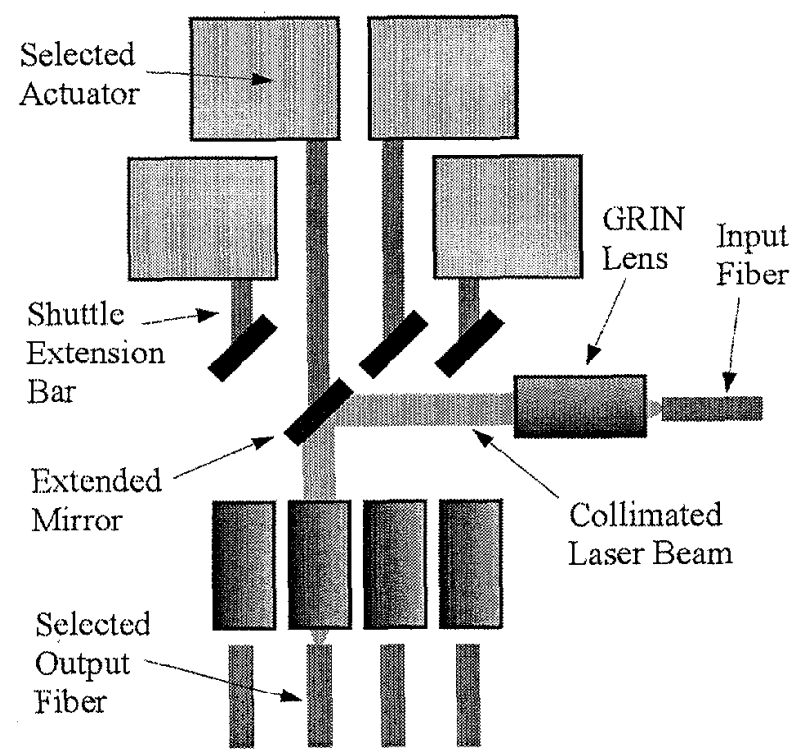

Figure 1. Schematic diagram showing a IX4 optical switch. Light leaving the input fiber is collimated by a GRIN lens and travels left until it intercepts an extended mirror and is focused onto an output fiber.

This paper describes a linear actuator, fabricated using deep reactive ion etching, that has been optimized for use in optical switching systems such as that shown in Figure 1. In an example application for an optical data storage system, the actuator must be as small as possible with the ability to move a mirror a total distance of $200 \mathrm{um}$ in less than $1 \mathrm{~ms}$ while using less than $150 \mathrm{~V}$.

\section{SWITCH DESIGN}

In the example data storage application, at least $80 \%$ coupling efficiency is required with switching speeds below $1 \mathrm{~ms}$. Few existing optical switches meet these specifications, and none are available that also fit within the $18 \mathrm{~cm}^{3}$ device volume. The proposed optical switch requires an actuator for each output fiber. A collimated laser beam passes down the switch's central axis, as shown in Figure 1. During operation, one actuator is extended while all others are retracted. A mirror attached to the actuator serves to redirect the laser beam into the selected output fiber. The mirror width should be twice the collimated beam width to reduce diffractive losses, and the actuator must have a total deflection that is at least the width of the mirror. The difficulty in designing largc-deflection actuators provides a motivation to reduce the collimated beam diameter. However, as the beam diameter is reduced, the distance over which the beam remains collimated is also reduced. The divergence of the optical beam results in a reduction 
in the achievable coupling efficiency. The proposed optical switch uses 250 um diameter GRIN lenses to produce a 100 um diameter collimated beam. The divergence of the beam over the length of the switch is then small enough allow up to $80 \%$ coupling efficiency.

\section{ACTUATOR DESIGN}

Several novel features facilitate the design of the largedeflection electrostatic actuators that are required for the proposed optical switch. As shown in Figure $2 \mathrm{a}$, conventional comb drives are typically symmetric about the translation axis, having four folded-beam suspension springs [6]. The proposed actuator is not symmetric about the translation axis, allowing it to consume only half the area of a traditional design. Since a one-sided design has half the mass, half the springs, and half the combs (see Figure 2b), both the suspension stiffness and the electrostatic force are reduced by a factor of two. Thus neither the resonant frequency nor the maximum static deflection are changed. Alternatively, a one-sided design may occupy the same area as a traditional comb-drive, but use the area more efficiently to achieve a larger stable forward deflection range. Note that in Figure $2 b$, the suspension has been placed exterior to the combs to improve the low rotational stiffness of the suspension shown in Figure $2 \mathrm{a}$.

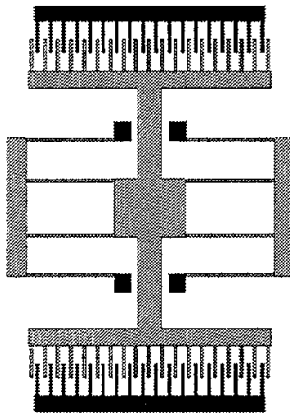

(a)

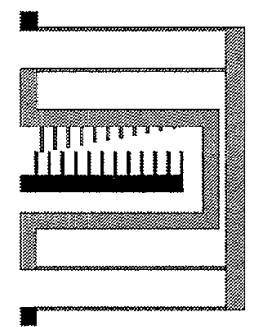

(d)

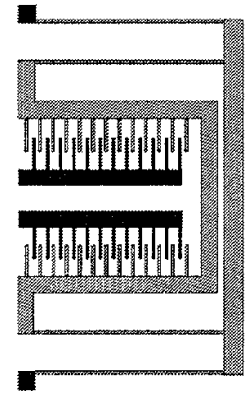

(b)

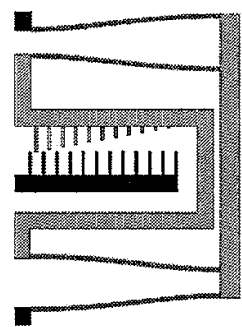

(e)

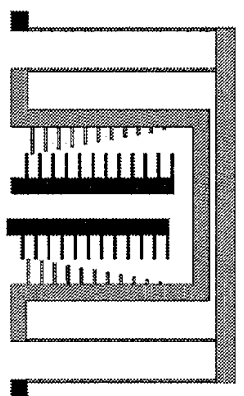

(c)

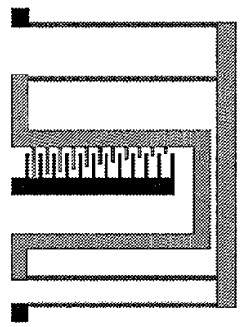

(f)
Figure 2. Schematic diagram of a traditional comb-drive actuator (a), a one-sided actuator (b), an actuator with linear engagement comb teeth (c), an actuator with a parallel-plate electrode (d), an actuator with a prebent suspension as-fabricated (e), and an actuator with a prebent suspension after forward deflection $(f)$.

In the proposed optical switch, the actuators are only required to fully extend or retract. Switching speed and positional accuracy may be increased by using mechanical stops to define the two actuator states. The actuator then drives the shuttle to press against either forward or rear crash-stops that are fixed to the substrate. The crash stops allow the electrostatic force versus the deflection profile to be customized. While the parametric design of nonrectangular comb teeth has been described $[4,7]$, a simpler way to modify the displacement dependence of the electrostatic force is to adjust the lengths of the individual comb teeth. By linearly increasing the length of the combs that form the moving electrode, the number of engaged comb teeth varies linearly with forward displacement (see Figure 2c). The forward electrostatic force is then

$$
F=\frac{(N-1) \varepsilon h x}{g x_{\max }} V^{2}+\frac{\varepsilon h}{2 g} V^{2},
$$

where $N$ is the number of comb teeth, $\varepsilon$ is the dielectric constant of the air gap, $h$ is the device thickness, $x$ is the forward displacement, $g$ is the electrostatic gap, and $V$ is the applied voltage. This linear engagement comb drive achieves the maximum forward deflection, $x_{\text {max }}$, at the same voltage as a corresponding traditional comb drive. However, with only half the electrostatic overlap area, the linear engagement design suffers less from electrostatic instability since it only generates half the detrimental parallel-plate forces between the sides of the comb teeth.

The use of mechanical stops to define the shuttle position also allows the design of an actuator with only one bank of opposing comb teeth (see Figure 2d). In the proposed actuator the motor is retracted 110 um by a set of comb teeth; it is extended by releasing the retracted shuttle and allowing it to glide freely to a set of mechanical stops 90 um in the extended direction, where it is captured by a pair of parallel plate electrodes. By using the back side of the fixed combs to form the fixed parallel plate, the resulting compact actuator (about $1 \mathrm{~mm}^{2}$ ) only requires a single electrical drive pad. The actuator changes state when the drive voltage is removed, and the new state is locked in when the voltage is reapplied

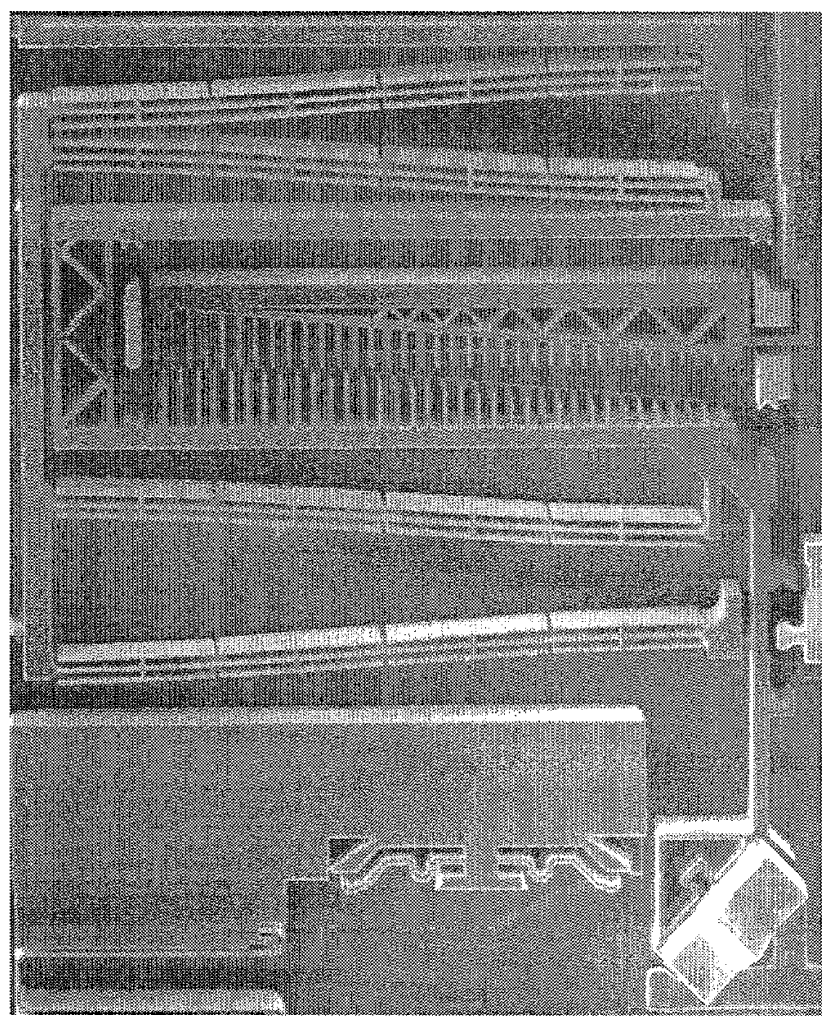

Figure 3. SEM image of the actuator used in the optical switch. The four " $H$ " shaped fins attached to each suspension beam help control the sidewall profile without changing the spring stiffness. 
Finally, with only one bank of opposing comb teeth, the folded-flexure suspension may be customized. The side stiffness, $k_{\text {side }}$, of a traditional folded-flexure suspension falls with the square of the forward displacement $[2,8]$ :

$$
k_{\text {side }}=\frac{E h w}{L}\left(\frac{3 x^{2}}{8 w^{2}}+1\right)^{-1},
$$

where $E$ is the Young's modulus of silicon, $x$ is the forward displacement, and $L, w$, and $h$ are the length, width, and thickness of the individual suspension beams. The situation may be dramatically improved by fabricating the suspension beams in an initially bent configuration, as shown in Figure $2 \mathrm{e}$. A cosine with amplitude $x_{p b} / 4$ is an admissible function for the initial bend profile. This effectively replaces $x$ by $\left(x-x_{p b}\right)$ in Equation 2. The side stiffness increases as the shuttle moves forward, reaching a maximum when the beams are straight at $x=x_{p b}$ (see Figure 2f). In a typical actuator of the proposed design, the use of prebent suspension beams increases the minimum side stability over the actuation range by a factor of thirty. A SEM of an actuator similar to that of Figure $2 \mathrm{e}$ is shown in Figure 3 . Note that a separately fabricated mirror has been attached to a mirror holder on a shuttle extension bar.

\section{FABRICATION}

All of the front-end wafer processing for the actuators and optical switches was carried out at TRW Lucas NovaSensor, in Fremont, California. The five masks required for the process were provided to TRW Lucas NovaSensor and the parts were delivered in wafer form. The wafers were then tested and diced prior to assembly and packaging. The simple fabrication process kept performance variations across 4 wafers (more than 500 switches) below $5 \%$, with a defect rate of only $7 \%$. While the proprietary details of the TRW Lucas NovaSensor process have not been disclosed, their generalized fabrication process has been described [9] (see Figure 4).

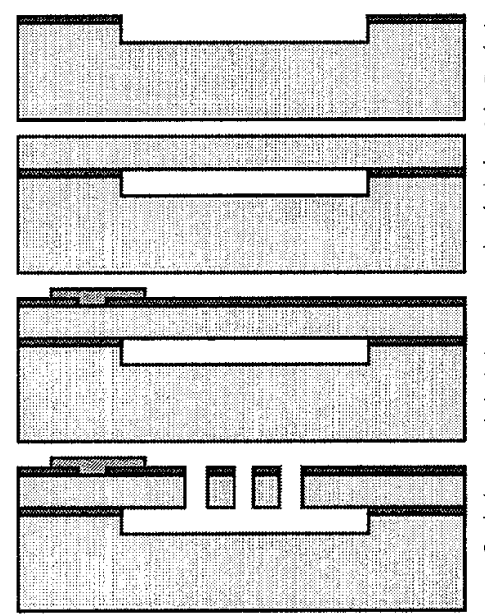

Etch shallow cavity in carrier wafer to create future movable areas.

Fusion bond device wafer to carrier; polish to final thickness.

Oxidize; open contact holes; deposit and pattern pad metal.

DRIE etch through device wafer.

Figure 4. Schematic diagram showing the fabrication process carried out at TRW Lucas Novasensor.

\section{ACTUATOR PERFORMANCE}

After fabrication, dicing, and assembly, the actuators (see Figures $2 \mathrm{e}$ and $2 \mathrm{f}$ ) were tested both statically and dynamically. The static response shown in Figure 5, was generated by increasing the drive voltage while measuring the forward deflection with an optical microscope at $1000 \mathrm{x}$ magnification. Unlike a traditional comb drive. the displacement of a linear engagement design is not directly proportional to the square of the voltage. The maximum forward deflection is defined by the placement of the forward crash stops.

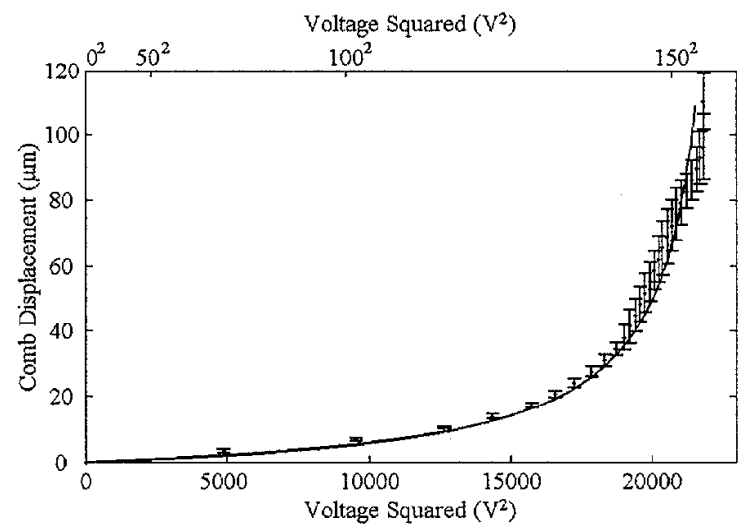

Figure 5. Comparison of the measured (circles) and predicted (line) quasi-static response of a linear-engagement actuator with a pre-bent suspension.

A laser vibrometer was used to quantify the resonant behavior of the actuators. Figure 6 shows the swept sine frequency response of an actuator and the response predicted by a Simulink ${ }^{\mathrm{TM}}$ model of the actuator as a 2 DOF, second-order system. The fundamental resonance occurred at $995 \mathrm{~Hz}$ with a quality factor of 57 . The resonance at $4 \mathrm{kHz}$ results from the contrary motion of the shuttle and the suspension truss. These dynamic modes, as well as the additional mode at $9 \mathrm{kHz}$, match those predicted by a Mechanica ${ }^{\mathrm{TM}}$ finite clement model.

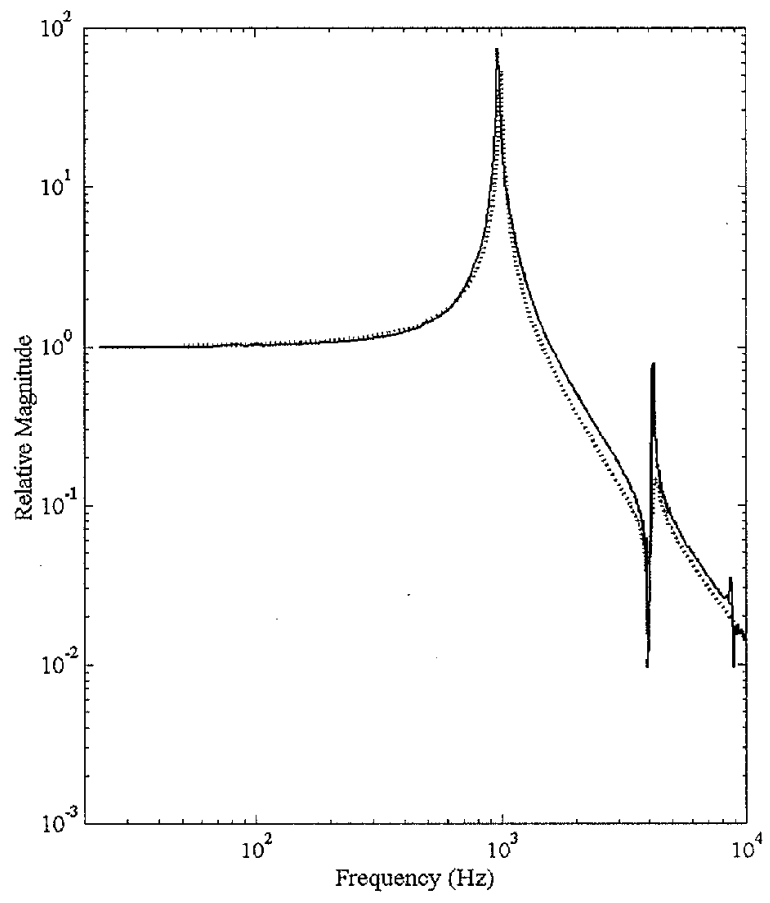

Figure 6. Measured and predicted frequency response magnitude. The solid line represents data gathered using a laser vibrometer. The dotted line represents the response predicted by $F E A$ and numerical models using Mechanica ${ }^{T M}$ and Simulink ${ }^{T M}$. 


\section{SWITCH PERFORMANCE}

Optical switches from $1 \times 2$ to $1 \times 16$ have been fabricated, and a portion of an assembled switch is shown in the SEM of Figure 7. Additional polarization detection optics are present in this particular switch to facilitate its use in an optical data storage system.

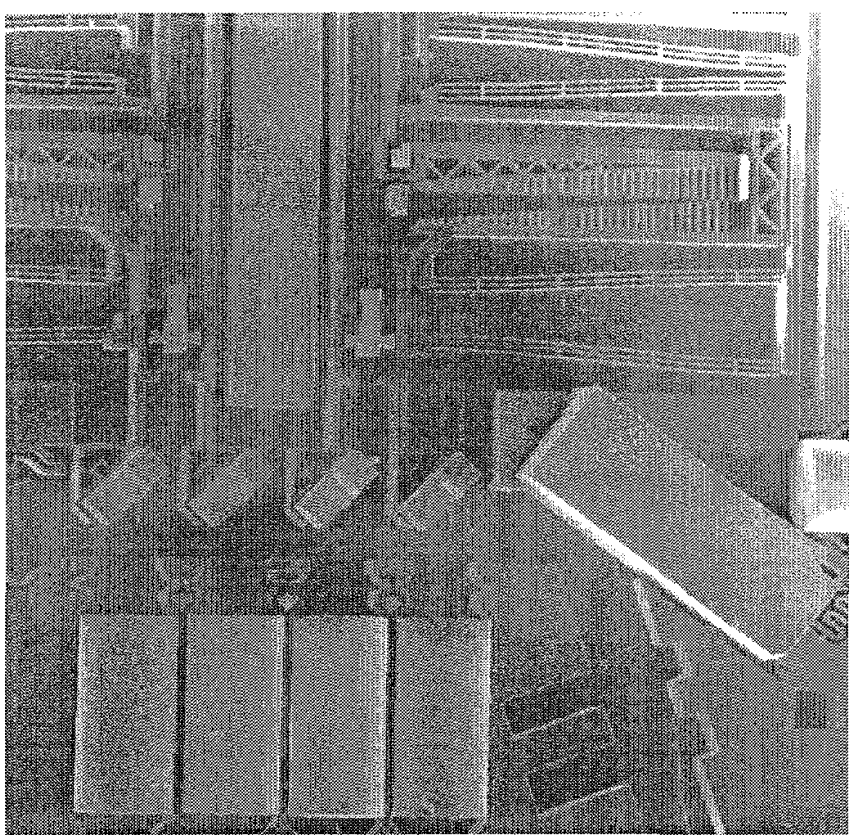

Figure 7. SEM view of a portion of a partially assembled optical switch showing the actuators, the inserted mirrors, and the cylindrical GRIN lenses held in place with integral alignment springs. Additional polarization detection optics are present for use in a data storage system.

In order to achieve the highest optical efficiency, separately fabricated mirrors are inserted into holders in the linear actuators. These mirrors have high reflectivity with minimal scattering loss, which is an improvement over approaches that use the etched sidewall as the mirror surface [10]. Switching losses of less than 1 $\mathrm{dB}$ at a wavelength of $650 \mathrm{~nm}$ (and less than $0.5 \mathrm{~dB}$ at $1550 \mathrm{~nm}$ ) have been demonstrated using single mode optical fibers.

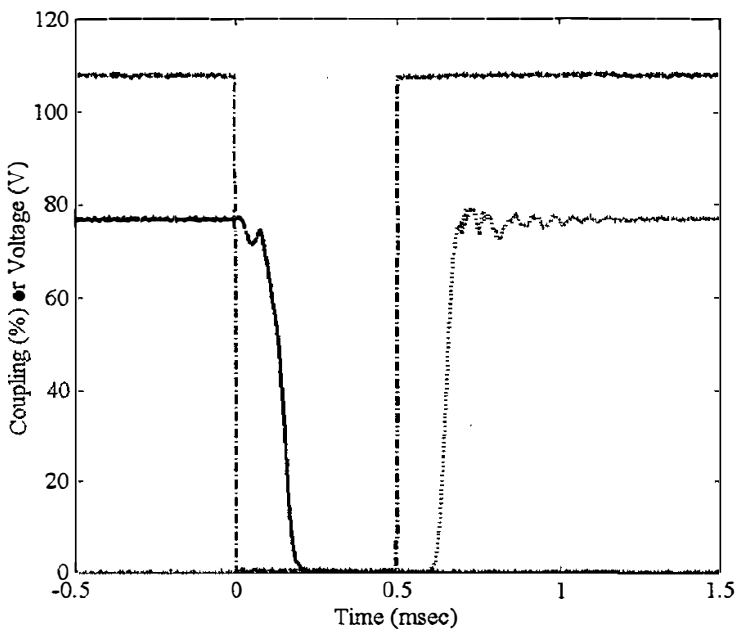

Figure 8. Measured drive voltage (dash-dot line) and coupling efficiency as light is switched from one fiber (solid line) to another (dotted line).
The coupling efficiency and switching speed were measured using a high-speed power meter and a digitizing oscilloscope. Since the resonant frequency of the actuators is typically $1 \mathrm{kHz}$, the switching time is slightly greater than $0.5 \mathrm{~ms}$, as shown in Figure 8 . Note that the actuator begins to change state when the drive voltage is removed, and the new state is locked in after the voltage is reapplied. The previously selected actuator and the newly selected actuator change state simultaneously, due to the same voltage signal.

\section{SUMMARY}

Through substantial improvements in design, comb-drive actuators consuming only $1 \mathrm{~mm}^{2}$ have been demonstrated with stable, static deflections of 200 um and switching speeds of less than $1 \mathrm{~ms}$. The simple, high-yield, fabrication process has produced consistent performance that closely matches FEA and numerical models of the electrostatic actuators. These actuators have been utilized in a compact optical design that features high-speed, lowloss switching.

\section{ACKNOWLEDGEMENTS}

The authors would like to thank Kathy Jackson, Pei-Fei Li, and Chris Tan at Seagate Research, and Sam Wong at TRW Lucas NovaSensors for their help with fabrication, assembly, and testing.

\section{REFERENCES}

[1] J.H. Jerman, et. al., "Electrostatic Microactuator and Method for Use Thereof", U.S. Patent 5,998,906 (1999).

[2] R. Legtenberg, et. al., "Comb-Drive Actuators for Large Displacements", J. Micromech. Microeng., 6 (1996), p. 320-329.

[3] C. Marxer, et. al., "An Electrostatic Actuator with Large Dynamic Range and Linear Displacement-Voltage Behavior for a Miniature Spectrometer", Transducers '99, p. 786-789.

[4] J. Mohr, et. al., "Micro Optical Switching by Electrostatic Linear Actuators with Large Displacements", Transducers '93, p. 120-123.

[5] C. S.-B. Lee, et. al., "Multiple Depth, Single Crystal Silicon MicroActuators for Large Displacement Fabricated by Deep Reactive Ion Etching", 1998 Solid-State Sensor and Actuator Workshop, Hilton Head, SC, p. 45-50.

[6] W. Tang, et. al., "Laterally Driven Polysilicon Resonant Microstructures", Sensors and Actuators, 20, p. 25-32, 1989.

[7] W. Ye, et. al., "Optimal Shape Design of an Electrostatic Comb Drive in Microelectromechanical Systems", J. Microelectromechanical Systems, 7 (1998), p. 16-26.

[8] J. Grade, "Large-Deflection, High-Speed, Electrostatic Actuators for Optical Switching Applications", Ph.D. dissertation, Stanford University, 1999.

[9] E. Klaassen, et. Al., "Silicon Fusion Bonding and Deep Reactive Ion Etching; A New Technology for Microstructures", Sensors and Actuators, 52 (1996), p. 132-139.

[10] C. Marxer, et. al., "Vertical Mirrors Fabricated by Reactive Ion Etching for Fiber Optical Switching Applications", 10th Int. Workshop on Micro Electro Mech. Sys., p. 49-54. 